\title{
ORIGINAL ARTICLE \\ Sitting balance and limits of stability in persons with paraplegia
}

\author{
P Serra-Añó ${ }^{1}, M$ Pellicer-Chenoll ${ }^{2}$, X Garcia-Massó ${ }^{2}$, G Brizuela ${ }^{2}$, C García-Lucerga ${ }^{1}$ and L-M González ${ }^{2}$
}

Study Design: Cross-sectional, observational study of paraplegic and able-bodied persons.

Objectives: The aim of the study was to analyse the temporal and frequency domains of seated balance to better understand nervous system control in equilibrium in persons with spinal cord injury ( $\mathrm{SCl}$ ) and to explore their centre of pressure (CoP) limits before experiencing a fall.

Setting: University of Valencia, Spain.

Methods: Static and dynamic seated balance were assessed in 24 paraplegic persons divided into two groups: low paraplegia group (LP) and high paraplegia group (HP), and 24 healthy volunteers with an extensiometric force plate. Two types of tests were performed: a static test (ST), where data signal was analysed by temporal and frequency domains, and a stability limit test (SLT), where different stability limits were calculated.

Results: The paraplegic group revealed lower static postural control in both domains in most of the parameters analysed compared with the control group (CG). Similar results were obtained with regard to the SLT, showing differences in the three parameters analysed between the $\mathrm{CG}$ and $\mathrm{SCl}$ groups.

Conclusion: Posturographic assessment in ST was useful to explore nervous system control in equilibrium in this population, presenting a decreased balance in paraplegic groups and an altered pattern in the sensorial and cerebellum bands compared with able-bodied individuals. Furthermore, SLT indicated less movement control of the CoP in paraplegic groups, which may influence the performance of their daily activities.

Spinal Cord (2013) 51, 267-272; doi:10.1038/sc.2012.148; published online 27 November 2012

Keywords: spinal cord injury; level of injury; trunk control; centre of pressure; power spectral density

\section{INTRODUCTION}

Sitting balance maintenance is impaired in individuals with complete spinal cord injury (SCI) due to the impaired functionality of the muscles primarily responsible for postural control. ${ }^{1}$ Other mechanisms, like normal postural synergies and sensory-motor integration of the lower limbs and trunk, are also impaired. However, it has been proposed that following an SCI, a gradual development of specific motor synergies for balance control in sitting involving even non-postural muscles ${ }^{2-5}$ would lead to reorganisation of the individual's balance control system. . $^{1,6}$

These strategies are essential, as sitting is one of the most fundamental activities of daily living for persons with $\mathrm{SCI}^{7,8}$ and this ability determines their level of independence for many activities of daily living. ${ }^{9}$ For this reason, an appropriate measurement of the sitting posture and sitting movements is imperative for persons with paraplegia.

Previous studies have analysed dynamic sitting balance by means of upper extremity reaching tasks, in which targets were placed at different distances from the patients to explore parameters such as reaction time. ${ }^{1,10}$ However, these studies did not account for the participants' maximum range before a fall.

Other studies have analysed the static sitting position with a force plate by placing their participants in a long-sitting position. ${ }^{11}$ Although this position is common among persons with SCI, this population spends most of the day seated in a wheelchair. ${ }^{12}$ Furthermore, this previous research assessed balance in the temporal domain. However, frequency analysis could provide important information about the different contributors' patterns to the postural control.

According to Bizid et al., ${ }^{13}$ in the frequency analysis of postural control, the total spectral energy is distributed into three frequency bands depending on the type of somatic regulation: action of the sensory systems (visual and vestibular) is represented by low frequencies $(0-0.5 \mathrm{~Hz})$; regulation of the cerebellum corresponds to intermediate frequencies $(0.5-2 \mathrm{~Hz})$; and proprioceptive regulation is expressed by high frequencies $(>2 \mathrm{~Hz})$.

The aim of the study was to analyse the temporal and frequency domains of seated balance by means of a seated-static test (ST) simulating the common position in a wheelchair, to better understand nervous system control in equilibrium in persons with SCI, and to explore their centre of pressure $(\mathrm{CoP})$ limits before experiencing a fall with a stability limit test (SLT).

\section{MATERIALS AND METHODS \\ Participants}

In total, 24 persons with paraplegia (21 men, 3 women) were assessed. In this group mean age was $40.30(8.81)$ years, the mean weight was $77.29(15.17) \mathrm{kg}$, the mean seated height was $1.01(0.58) \mathrm{m}$ and the mean injury duration was 
14.2 (8.70) years. Their injury levels ranged from the fourth thoracic (T4) to the first lumbar (L1) vertebra. For the paraplegic group, the selection criteria, according to the American Spinal Injury Association Impairment Scale (AIS), included: paraplegia grade 'A' (represent complete impairment, that is, no sensory or motor function in the S2-S4 segment), and paraplegia grade ' $\mathrm{B}$ ' (sensory incomplete, no motor function below the neurological level and includes the sacral segments), ${ }^{14}$ lasting at least 12 months, an absence of pelvic obliquity and/or scoliosis and independence in performing transfers. The presence of pressure ulcers and vestibular disorder or pronounced visual deficiency and incomplete motor and sensory function (grade ' $\mathrm{C}$ ' according to the AIS $)^{14}$ were considered excluding criteria for participation in the study. This paraplegic group was divided into two subgroups: high paraplegia (HP), in which the level of injury was above T9, and low paraplegia (LP), in which the level of injury was equal to or below T9. ${ }^{1}$

The control group (CG) was comprised of 24 healthy persons (14 men, 10 women) with a mean age of 27.67 (12.62) years, a mean weight of 69.74 $(14.03) \mathrm{kg}$ and a mean seated height of $0.92(0.37) \mathrm{m}$. All subjects completed this study without adverse effects. Groups were matched for weight and height. All participants provided written informed consent, and all procedures were conducted in accordance with the principles of the World Medical Association's Declaration of Helsinki and were approved by the ethical committee of the University of Valencia.

\section{Experimental devices and procedures}

One force plate (DINASCAN, IBV, Valencia, Spain) was used to assess the sitting balance of each participant. On the platform, a specially made $70-\mathrm{cm}$ height stool without a backrest and with the same base dimensions as the support base of the platform was installed. This stool had adjustable footrests to assure $90^{\circ}$ knee flexion, with the aim of accurately representing the sitting posture of paraplegic individuals in a wheelchair. Moreover, all participants were under close supervision of the clinician at all times and wore a safety harness in order to prevent any possible harm while performing the tests (Figure 1).

Subjects were asked to sit centred on the stool, with the sacrum $1 \mathrm{~cm}$ from the posterior side of the stool. In order to familiarise study participants with the tests, procedures were practiced for $10 \mathrm{~min}$, followed by a 2-min-period rest, before commencing the assessment. The first part of the test included a ST in two different conditions: eyes opened (EO) and eyes closed (EC). Individuals had to maintain their sitting position on the stool for $30 \mathrm{~s}$ with their arms relaxed along the trunk. Two repetitions of each condition were conducted with $30 \mathrm{~s}$ rest period in between.

In the second part, subjects performed a SLT. Beginning from the same position as in the previous test, individuals tried to shift their CoP, displacing their trunk, as far as possible to address different targets that appeared on the

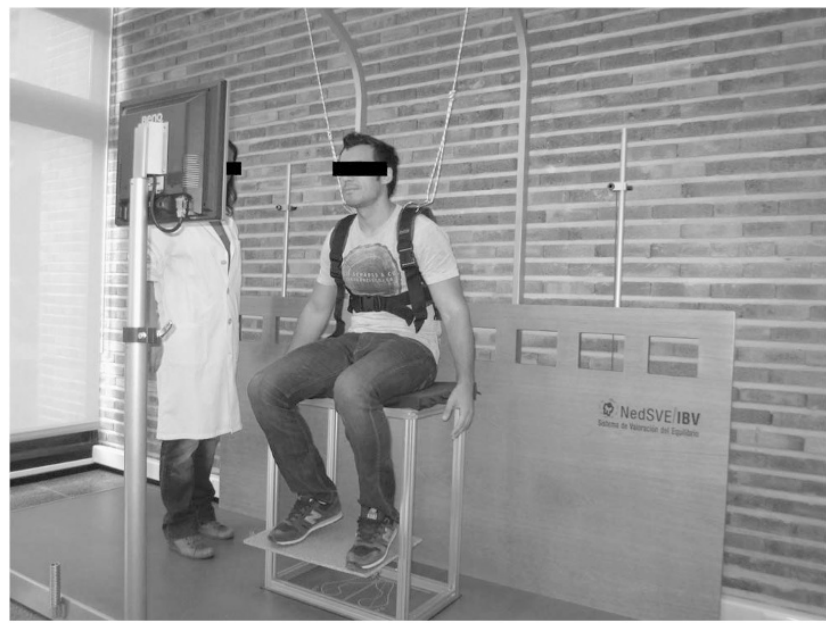

Figure 1 The experimental set-up for balance testing. The stool is firmly attached to the extensiometric force plate. computer screen placed at the height of their eyes. These targets were placed in four directions (forward, backward, right and left). They performed an isolated repetition of the SLT to avoid fatigue and prevent the occurrence of possible sore pressures.

\section{Data analysis}

Signals were recorded at a frequency of $40 \mathrm{~Hz}$ using an amplified analogue-todigital converter. The CoP displacement data were obtained considering that the CoP was applied on the platform at a height of $70 \mathrm{~cm}$. The Matlab $2010 \mathrm{~b}$ (Mathworks Inc., Natick, MA, USA) programme was used to condition the signals and to calculate the stabilometry variables. ST signals were filtered digitally using a fourth-order Butterworth low-pass filter with a 6-Hz cutoff frequency. The first and last $5 \mathrm{~s}$ of each attempt were discarded whenever they appeared unstable.

For the ST, in the temporal domain, the signal amplitude was calculated in the anterior-posterior and medial-lateral directions using the root mean square (RMS) and the total range of movement of the CoP (range). The 95\% confidence ellipse area (Area-CE) was also calculated.

In the frequency domain, power spectral density was calculated between 0.15 and $6 \mathrm{~Hz}$ (resolution $=0.025 \mathrm{~Hz}$ ) using the Fast Fourier transform algorithm. The total energy of the spectrum and the median power frequency were estimated. The percentage distribution of the spectral energy in three frequency bands was also calculated: ${ }^{13}$ low frequency, $0.15-0.5 \mathrm{~Hz}$; medium frequency, $0.5-2 \mathrm{~Hz}$; high frequency, $2-6 \mathrm{~Hz}$.

For SLT, three statistical parameters were calculated in the four directions tested: forward, right, backward and left. One statistical parameter was the maximal CoP displacement (CoPmax), which represents the furthest target achieved in each of the directions.

Although CoPmax indicates the maximal limits of stability, the maximal target is typically reached only momentarily. To establish the farthest point of the $\mathrm{CoP}$ in which individuals remain more time, the efficient CoP displacement (CoPeffi) was calculated. For this purpose, the distance from the initial CoP to CoPmax was halved. The furthest half was then divided into 1-mm areas, and the amount of time the CoP remained in each of these areas was calculated. The point at which the $\mathrm{CoP}$ of each individual mostly remained was considered the CoPeffi.

Finally, a parameter of instability of the pathway was calculated (CoPins). This parameter assessed the normalised total excursion of the CoP covered to achieve the limit of stability by the CoPmax. Figure 2 illustrates this analysis in one individual with paraplegia.

\section{Statistical analysis}

Statistical analysis was performed using PASW v.18 (SPSS Inc., Chicago, IL, USA). All dependent variables complied with the assumption of normality (Shapiro-Wilks normality test). Standard statistical methods were used to obtain the mean and the s.d. of the mean.

For possible effects of group, condition and direction on the ST variables, multivariate analyses of variance were applied. As direction was not a possible factor in Area-CE, this variable was analysed by means of a mixed analyses of variance. For possible effects of group and direction on the SLT variables, multivariate analyses of variance were used. Pairwise comparisons were performed using the Bonferroni approach. The level of statistical significance was set at $P<0.05$.

\section{RESULTS}

For the ST, the main effect of the group $\left(\mathrm{F}_{12,82}=7.93, P<0.001\right.$, $\left.\eta_{\mathrm{p}}^{2}=0.54\right)$, the main effect of the condition $\left(\mathrm{F}_{6,40}=15.11, P<0.001\right.$, $\left.\eta_{\mathrm{p}}^{2}=0.69\right)$ and the main effect of the direction $\left(\mathrm{F}_{6,40}=101.15\right.$, $\left.P<0.001, \eta_{p}^{2}=0.94\right)$ were significant in the multivariate contrasts. There was also an interaction effect between group and direction $\left(\mathrm{F}_{12,82}=5.0, P<0.001, \eta_{\mathrm{p}}^{2}=0.42\right)$ and between group and condition $\left(\mathrm{F}_{12,82}=3.871, P<0.001, \eta_{\mathrm{p}}^{2}=0.36\right)$.

In the anterior-posterior direction, RMS and range were lower in CG than in LP and HP, both during EO and EC $(P<0.05)$. Regarding 

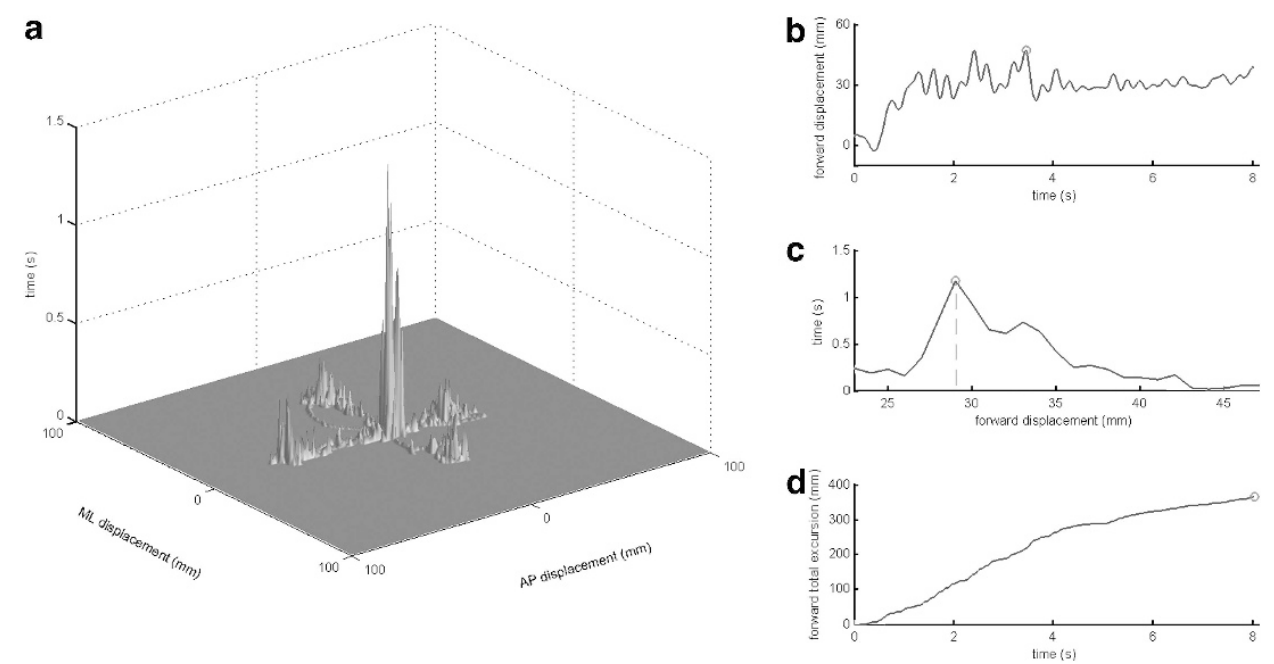

Figure 2 Stability limits test in one of the paraplegic subjects (panel a) and an example of the data analysis conducted on the forward direction of movement (panels b-d). Panel (a) illustrates an example of the stability limits over the four directions as a function of time. In panel (b), CoPmax is represented by a circumference. In panel (c), the circumference illustrates the point of the pathway in which the furthest stability limit is maintained for the longest time, and the broken vertical line illustrates the efficient CoP displacement (CoPeffi). In panel (d), the circumference represents the maximal total excursion. This parameter divided by CoPmax results in the instability of the pathway (CoPins). Values for the example are $46.93 \mathrm{~mm}, 29 \mathrm{~mm}$ and 7.72 (no units), for CoPmax, CoPeffi and CoPins, respectively.

Table 1 Results of the seated test in the medial-lateral parameters

\begin{tabular}{|c|c|c|c|c|c|c|c|c|c|c|c|c|c|c|c|}
\hline & \multicolumn{2}{|c|}{$C G$} & \multicolumn{2}{|r|}{$L P$} & \multicolumn{2}{|c|}{$H P$} & \multicolumn{2}{|c|}{$C G-L P$} & \multicolumn{2}{|c|}{ CG-HP } & \multicolumn{2}{|c|}{$H P-L P$} & \multicolumn{3}{|c|}{ Condition } \\
\hline & $E O$ & $E C$ & $E O$ & $E C$ & $E O$ & $E C$ & $E O$ & $E C$ & $E O$ & $E C$ & $E O$ & $E C$ & $C G$ & $L P$ & $H P$ \\
\hline RMS (mm) & $0.40(0.10)$ & $0.44(0.14)$ & $1.06(0.42)$ & $1.63(0.83)$ & $1.34(0.55)$ & $2.27(0.84)$ & * & * & * & * & & * & & * & * \\
\hline Range (mm) & $3.52(0.46)$ & $3.41(0.80)$ & $8.88(6.55)$ & $12.64(7.80)$ & $9.34(4.59)$ & $17.26(10.65)$ & * & * & * & * & & & & * & * \\
\hline Energy $\mathrm{mm}^{2} \cdot \mathrm{Hz}^{-1}$ & $5.32(2.30)$ & $5.92(3.25)$ & $48.71(52.67)$ & $103.13(104.70)$ & $54.78(37.71)$ & 147.37 (111.09) & * & * & * & * & & & & * & * \\
\hline Low-frequency band (\%) & $21.32(9.62)$ & $24.76(10.04)$ & $28.50(11.50)$ & $42.57(18.19)$ & $36.57(11.55)$ & $46.43(15.50)$ & & * & * & * & & & & * & * \\
\hline Intermediate frequency band (\%) & $70.01(8.17)$ & $66.06(8.21)$ & $63.62(10.13)$ & $52.12(15.93)$ & $56.20(11.58)$ & $45.84(12.59)$ & & * & * & * & & & & * & * \\
\hline High-frequency band (\%) & $8.66(3.04)$ & $9.18(4.11)$ & $7.87(6.98)$ & $5.32(3.53)$ & $7.22(4.86)$ & $7.73(5.30)$ & & & & & & & & & \\
\hline Med power frequency $(\mathrm{Hz})$ & $1.08(0.20)$ & $0.95(0.22)$ & $0.88(0.23)$ & $0.65(0.24)$ & $0.74(0.26)$ & $0.59(0.24)$ & & * & * & * & & & * & * & * \\
\hline
\end{tabular}

Abbreviations: CG, control group; LP, low paraplegic individuals; HP, high paraplegic individuals; EO, under eyes open; EC, eyes in closed conditions; RMS, root mean square.

" $P<0.05$. Comparison of the medial-lateral parameters of the test were computed for a group of healthy adults. 'Condition' refers to visual information in each test within every group. The values are expressed as the mean (s.d.).

energy, CG showed lower values than LP during EO $(P<0.05)$ and lower values than HP in both conditions $(P<0.05)$.

Concerning energy bands, the lower band presented a higher contribution in the postural control in HP than in CG in EO $(P<0.05)$. However, both paraplegics groups showed a lower contribution of the intermediate band than CG, performing the test with EC $(P<0.05)$.

In the medial-lateral direction, RMS, range and energy were lower in CG than in both paraplegic groups during both conditions $(P<0.05)$. Furthermore, RMS was greater in HP than in LP when individuals performed the test with EC $(P<0.05)$.

With respect to the frequency bands, the contribution of the lower band was greater in HP than in CG during both conditions. This lower band also presented greater values in LP than in CG, but only during EC $(P<0.05)$. As occurred in the anterior-posterior direction, the contribution of the intermediate frequency was lower in HP than in CG during both conditions $(P<0.05)$ and it was also lower in LP than in CG during EC $(P<0.05)$. Finally, HP showed a lower median frequency than CG during both conditions $(P<0.05)$ and it was also lower in LP compared with CG during EC $(P<0.05)$. Tables 1 and 2 display descriptive statistics and post-hoc analyses of the ST, in the three groups, and the two conditions.
The analyses of variance showed a main effect of group $\left(\mathrm{F}_{2,44}=22.92, P<0.001, \eta_{\mathrm{p}}^{2}=0.51\right)$ in Area-CE. Area-CE was lower in the CG than in the HP when the eyes were open. In the in the EC condition, CG showed lower Area-CE than LP and HP. Moreover, HP presented a large Area-CE than LP in EC condition (Figure 3).

Regarding SLT, there was a significant main effect of the groups $\left(\mathrm{F}_{6,88}=15.394, \quad P<0.001, \quad \eta_{\mathrm{p}}^{2}=0.51\right)$ and of the direction $\left(\mathrm{F}_{9,37}=2.18, P=0.046, \eta_{\mathrm{p}}^{2}=0.35\right)$, although the interaction between group and direction of the CoP was not significant $\left(\mathrm{F}_{18,76}=1.144\right.$, $P=0.329$ ). Table 3 displays the descriptive data and post-hoc results of the parameters obtained in the SLT in each group and each direction. The CG showed lower CoPins than the LP and HP $(P<0.05)$ in all directions. In addition, the values of CoPins in the HP were higher than those of the LP in right and left directions $(P<0.05)$. Finally, the CG showed higher CoPmax and CoPeffi values than the LP and HP $(P<0.05)$ in all directions.

\section{DISCUSSION}

The assessment conducted in our study reveals that postural control in a sitting position was substantially decreased among the paraplegic participants compared with the control subjects in both the temporal and frequency domains. This disabling situation is exacerbated in 
Table 2 Results of the seated test in the anterior-posterior parameters

\begin{tabular}{|c|c|c|c|c|c|c|c|c|c|c|c|c|c|c|c|}
\hline & \multicolumn{2}{|c|}{$C G$} & \multicolumn{2}{|c|}{$L P$} & \multicolumn{2}{|c|}{$H P$} & \multicolumn{2}{|c|}{$C G-L P$} & \multicolumn{2}{|c|}{$C G-H P$} & \multicolumn{2}{|c|}{$H P-L P$} & \multicolumn{3}{|c|}{ Condition } \\
\hline & $E O$ & $E C$ & $E O$ & $E C$ & $E O$ & $E C$ & $E O$ & $E C$ & $E O$ & $E C$ & $E O$ & $E C$ & $C G$ & $L P$ & $H P$ \\
\hline RMS (mm) & $0.84(0.15)$ & $0.86(0.25)$ & $2.15(1.07)$ & 3.35 (1.69) & $2.52(0.96)$ & $4.60(2.35)$ & * & * & * & * & & & & * & * \\
\hline Range (mm) & $2.17(0.69)$ & $2.17(0.66)$ & $5.76(2.80)$ & $7.60(3.83)$ & $6.29(2.69)$ & $10.40(3.93)$ & * & * & * & * & & * & & * & * \\
\hline Energy $\mathrm{mm}^{2} \cdot \mathrm{Hz}^{-1}$ & $9.38(2.64)$ & $7.17(3.91)$ & $80.08(96.67)$ & $160.12(179.20)$ & $77.21(70.00)$ & $320.55(343.18)$ & * & & * & * & & & & & * \\
\hline Low-frequency band (\%) & $37.64(9.80)$ & $36.22(9.48)$ & $41.22(11.49)$ & $49.21(16.80)$ & 41.38 (13.30) & $48.96(14.56)$ & & & & * & & & & * & * \\
\hline Intermediate frequency band (\%) & $48.10(7.73)$ & $48.76(6.25)$ & $46.58(7.34)$ & 41.29 (13.38) & $45.58(9.04)$ & $37.63(7.55)$ & & * & & * & & & & & * \\
\hline High-frequency band (\%) & $14.26(5.38)$ & $15.02(4.74)$ & $12.20(8.74)$ & $9.49(7.67)$ & $13.05(9.42)$ & 13.41(12.46) & & & & & & & & & \\
\hline Med power frequency $(\mathrm{Hz})$ & $0.81(0.23)$ & $0.81(0.21)$ & $0.72(0.27)$ & $0.59(0.26)$ & $0.71(0.37)$ & $0.67(0.43)$ & & & & & & & & & \\
\hline
\end{tabular}

Abbreviations: CG, control group; LP, low paraplegic individuals; HP, high paraplegic individuals; EO, under eyes open; EC, eyes in closed conditions; RMS, root mean square.

* $P<0.05$. Comparison of the anterior-posterior parameters of the test were computed for a group of healthy adults. 'Condition' refers to visual information in each test within every group. The values are expressed as mean (s.d.).

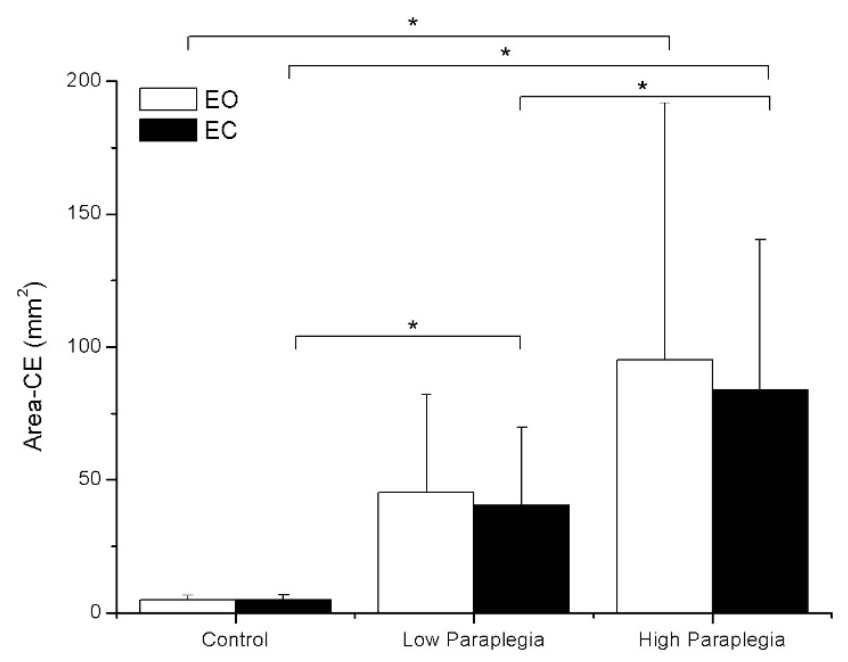

Figure 3 Area-CE as a function of group and condition. ${ }^{*} P<0.05$. CG, healthy adults; LP, low paraplegic individuals; HP, high paraplegic individuals; EO, eyes open; EC, eyes closed. The values are expressed as the mean (s.d.).

patients with higher level of injury. SCI patients interact with their environment from a sitting position, both to maintain a static posture and to achieve certain items. The ability to perform both tasks can be quantified through posturographic static and dynamic tests like those conducted in our study. Although there are previous studies in which balance assessment was conducted, ${ }^{15}$ logistical and methodological difficulties occurred in the development of the valuation, which makes it necessary to consider the protocols employed.

We attempted to design an experiment with the greatest possible external validity employing a sitting position. To our knowledge, this population spends most of their daily time seated in a wheelchair. Thus, the subjects were placed on a stool with their feet over footrests to avoid pressure on the popliteal fossa, which triggers spasticity, and to provide security in the test position. This position was maintained by the majority of participants. A large number of participants performed the test in a C-shape position as they were unable to activate the back extensors muscles. Only a few subjects stayed over the stool in a relaxed upright position. Measurements were repeated on a few occasions, on participants who could not maintain the position due to their higher level lesions.

The principal advantage obtained from the assessment protocols applied is the opportunity to quantify balance in as real a situation as possible. Consequently, this could allow the use of our results not only to researchers in the field, but also to clinical professionals.
Several important results have been obtained in this study for understanding the phenomena underlying the control of balance in paraplegics. The analysis of the ST in the temporal domain revealed significant statistical differences in the main effects analysed and in the interaction between visual condition and group.

Both SCI groups presented decreased postural control in the temporal domain when the test was performed with the EC. This pattern was not obtained in the CG, possibly because this test is not demanding enough for individuals who maintain a correct integration of the mechanisms involved in postural control. ${ }^{16}$ The results obtained in the temporal domain were reiterated in the frequency domain.

Previous studies ${ }^{17}$ analysing the median frequency of the acceleration of the CoP show that it is lower in paraplegics than in CG, as it is seen in our results. However, there are no published studies presenting data energy in the three different bands of the spectrum of the CoP displacements ${ }^{13}$ in persons with paraplegia. Nevertheless, this type of analysis is useful for evaluating postural disturbances caused by specific diseases or conditions as it has been seen in previous studies. ${ }^{18-20}$

In the test performed with EC, the SCI subjects demonstrated a significant increase in the contribution of the low-frequency band. Thus, the suppression of visual information may increment the dependency of vestibular information. ${ }^{13}$ Obviously, changes in one band will affect the percentages in the others. Therefore, in the present study, the intermediate frequency with EC decreased, while the high band remained stable.

After explaining the analysis of the effects according to visual condition, differences in postural control are assessed according to group (HP, LP and CG). As presented in the results of this study, there were significant differences between the CG and both SCI groups in most of the parameters analysed, as has also been reported in previous studies. ${ }^{11}$ It seems reasonable to assume that the control individuals receive a greater quantity of proprioceptive signalling, as they have more functional motor pathways.

Among all parameters, only RMS and Area-CE indicated significant differences between the paraplegic groups. This result may be due to the fact that level of injury is not always a strong prognosticator of stability, ${ }^{15}$ as many factors can influence balance, like skill, experience, tight muscles, multilevel fusion surgery, spasticity, etc. Furthermore, the classification of the injury level employed the American Spinal Injury Association Impairment Scale, ${ }^{14}$ which does not allow differentiation of the motor level of the trunk. More advanced methodologies may establish a better localisation of the level of injury, facilitating a better differentiation between both groups and even establishing correlations among all the parameters with the level of injury. 
Table 3 Results of the seated-limits of stability test

\begin{tabular}{|c|c|c|c|c|c|c|c|}
\hline & & Control & $L P$ & $H P$ & $C G-L P$ & $C G-H P$ & $H P-L P$ \\
\hline \multirow[t]{4}{*}{ CoPmax (mm) } & $\mathrm{F}$ & 109.31 (25.39) & $37.97(12.50)$ & $33.43(19.24)$ & * & * & \\
\hline & $\mathrm{R}$ & $106.08(25.68)$ & $40.66(14.59)$ & 32.05 (15.68) & * & * & \\
\hline & $\mathrm{B}$ & $83.31(22.05)$ & $34.54(15.07)$ & $28.28(12.62)$ & * & * & \\
\hline & $\mathrm{L}$ & $106.18(28.88)$ & $37.98(13.11)$ & $33.34(13.02)$ & * & * & \\
\hline \multirow[t]{4}{*}{ CoPeffi (mm) } & $\mathrm{F}$ & $101.50(26.59)$ & $31.73(11.71)$ & $20.08(13.21)$ & * & * & \\
\hline & $\mathrm{R}$ & $94.42(30.05)$ & $35.82(13.50)$ & $23.62(10.02)$ & * & * & \\
\hline & B & $72.87(23.88)$ & 30.09 (12.28) & 19.38 (7.97) & * & * & \\
\hline & $\mathrm{L}$ & 97.67 (29.35) & $31.55(10.86)$ & $23.00(7.76)$ & * & * & \\
\hline \multirow[t]{4}{*}{ CoPins (no units) } & $\mathrm{F}$ & $1.10(0.27)$ & $1.43(2.50)$ & $5.95(2.57)$ & * & * & \\
\hline & $\mathrm{R}$ & $1.34 / 0.43)$ & $4.79(2.33)$ & $8.06(4.76)$ & * & * & * \\
\hline & B & $1.72(1.33)$ & $4.27(2.49)$ & $5.67(2.72)$ & * & * & \\
\hline & $\mathrm{L}$ & $1.39(0.34)$ & $4.54(1.25)$ & $6.78(3.35)$ & * & * & * \\
\hline
\end{tabular}

Abbreviations: F, forward; R, right; B, backward; L, left; CG, control group; LP, low paraplegic individuals; HP, high paraplegic individuals; CoPmax, maximal CoP displacement; COPeffi, efficient COP displacement; CoPins, instability of the pathway.

The values are expressed as mean (s.d.).

${ }^{*} P<0.05$. Parameters are presented for the four directions. The values are expressed as mean (s.d.).

The frequency domain analysis revealed statistical differences between the groups. Our results indicate that persons with SCI present reduced median frequency in medial-lateral displacements compared with CG, as was also reported in a previous study of children with myelomeningocele. ${ }^{18}$

Regarding frequency bands, there were significant differences between groups in the low and intermediate bands, mostly in the EC condition and medial-lateral displacements. The intermediate frequency band was lower in the SCI groups than in the CG, which could be due to the communication impairment between the cerebellum and spinal cord. Consequently, low band frequency (sensorial input) was greater in the SCI group, likely due to compensation for the impairment previously described.

Nevertheless, both SCI groups presented percentages of higher frequency bands similar to the CG. Different trends of proprioception could have been expected between the groups; however, this pattern did not occur. The small sample size of our study should be taken into consideration, as for higher frequency variable we could have committed a Type II error. However, this situation may be explained by the adaptations that occur from the onset of injury to the stable chronic phase. People with SCI develop motor synergies involving non-postural muscles for maintaining balance, ${ }^{2-5}$ including being able to activate all trunk muscles, even those below the injury level. ${ }^{2}$ Consequently, it is possible that those non-postural muscles supply the proprioceptive information of the impaired postural muscles. ${ }^{21}$

Therefore, further research is needed to provide more specific information about the mechanisms of postural control. It could also be useful to record the muscle activity of non-postural muscles with electromyography and brain activity with functional magnetic resonance imaging to assess the holistic behaviour of sitting balance in persons with SCI.

The SLT obtained information about participants' maximal displacement preceding a fall, their efficient range in which they reach their furthest limit of stability being stable and the instability during the pathway.

Although there were no significant differences in the interaction between the groups and the direction of the CoP, the main effect of group and direction were significant. There were differences in maximum range, efficient range and instability between the CG and both SCI groups. However, there were no differences in the displacement parameters between the two SCI groups. Although it could be due to the sample size, it is in agreement with a previous study. ${ }^{22}$

Only instability of the pathway presented differences between the HP and LP groups, and only in the lateral not forward and backward, directions. This result may have occurred because in the lateral direction, where the participants' support base is essentially their ischial tuberosities, the partial activation of abdominal muscles in the LP is helpful for better controlling the CoP movements in line with the targets.

In conclusion, posturographic assessment indicates lower balance among SCI patients in the temporal domain analysis. These results are useful for exploring nervous system control in equilibrium in this population, presenting a different pattern of low and intermediate frequency compared with healthy individuals. As functioning of the cerebellum pathways is impaired among SCI patients, an increase in sensorial contribution is consequently produced to assure postural control. Furthermore, the SLT indicated poorer movement control among the CoP of SCI groups, where paraplegics with higher levels of injury showed greater instability.

These findings imply some potential research and clinical applications. Our study provides a useful tool to explore postural control and reveals some information about central nervous system organisation, where a close interaction between biomechanical and neurophysiological evaluation is required. This holistic analysis could enable the possibility of discerning the level of injury and the reorganisation of specific motor synergies for the purpose of planning individualised treatments depending on the contributor impaired. In addition, it provides an instrument to control improvements of rehabilitation programs, which should aim, according to our findings, to consolidate the sensorial contributor and improve the proprioception above the injury level.

\section{CONFLICT OF INTEREST}

The authors declare no conflict of interest.

\section{ACKNOWLEDGEMENTS}

$\mathrm{XGM}$ is a Vali $+\mathrm{D}$ researcher in training with support from the Generalitat Valenciana. 
1 Seelen H, Potten Y, Huson A, Spaans F, Reulen J. Impaired balance control in paraplegic subjects. J Electromyogr Kinesiol 1997; 7: 149-160.

2 Bjerkefors A, Carpenter MG, Cresswell AG, Thorstensson A. Trunk muscle activation in a person with clinically complete thoracic spinal cord injury. J Rehabil Med 2009; 41 390-392.

3 Seelen H, Potten Y, Drukker J, Reulen J, Pons C. Development of new muscle synergies in postural control in spinal cord injured subjects. J Electromyogr Kinesiol 1998; 8: 23-34.

4 Potten Y, Seelen H, Drukker J, Reulen J, Drost M. Postural muscle responses in the spinal cord injured persons during forward reaching. Ergonomics 1999; 42: 1200-1215.

5 Kizony R, Raz L, Katz N, Weingarden H, Weiss PLT. Video-capture virtual reality system for patients with paraplegic spinal cord injury. J Rehabil Res Dev 2005; 42: 595-607.

6 Do M, Bouisset S, Moynot C. Are paraplegics handicapped in the execution of a manual task? Ergonomics 1985; 28: 1363-1375.

7 Janssen-Potten YJ, Seelen HA, Drukker J, Spaans F, Drost MR. The effect of footrests on sitting balance in paraplegic subjects. Arch Phys Med Rehabil 2002; 83: 642-648.

8 Hartshorn J. Spinal cord injury: concepts and management approaches. Am J Nurs 1988; 88: 921.

9 Dean CM, Shepherd RB. Task-related training improves performance of seated reaching tasks after stroke: a randomized controlled trial. Stroke 1997; 28: 722-728.

10 Seelen H, Potten Y, Adam J, Drukker J, Spaans F, Huson A. Postural motor programming in paraplegic patients during rehabilitation. Ergonomics 1998; 41: 302-316.

11 Shirado O, Kawase M, Minami A, Strax TE. Quantitative evaluation of long sitting in paraplegic patients with spinal cord injury1. Arch Phys Med Rehabil 2004; 85: 1251-1256.

12 Boswell-Ruys CL, Harvey LA, Barker JJ, Ben M, Middleton JW, Lord SR. Training unsupported sitting in people with chronic spinal cord injuries: a randomized controlled trial. Spinal Cord 2009; 48: 138-143.
13 Bizid R, Jully JL, Gonzalez G, François Y, Dupui P, Paillard T. Effects of fatigue induced by neuromuscular electrical stimulation on postural control. J Sci Med Sport 2009; 12 60-66.

14 Marino RJ, Barros T, Biering-Sorensen F, Burns SP, Donovan WH, Graves DE et al. International standards for neurological classification of spinal cord injury. J Spinal Cord Med 2003; 26: S50-S56.

15 Kamper D, Barin K, Parnianpour M, Reger S, Weed H. Preliminary investigation of the lateral postural stability of spinal cord-injured individuals subjected to dynamic perturbations. Spinal Cord 1999; 37: 40-46.

16 Massion J. Postural control systems in developmental perspective. Neurosci Biobehav Rev 1998; 22: 465-472.

17 Grigorenko A, Bjerkefors A, Rosdahl H, Hultling C, Alm M, Thorstensson A. Sitting balance and effects of kayak training in paraplegics. J Rehabil Med 2004; 36: 110-116.

18 Karlsson A, Norrlin S, Silander HC, Dahl M, Lanshammar H. Amplitude and frequency analysis of force plate data in sitting children with and without MMC. Clin Biomech 2000; 15: 541-545.

19 Giacomini PG, Alessandrini M, Evangelista M, Napolitano B, Lanciani R, Camaioni D. Impaired postural control in patients affected by tension-type headache. Eur J Pain 2004; 8: 579-583

20 Cabeza-Ruiz R, García-Massó X, Centeno-Prada RA, Beas-Jiménez JD, Colado JC, González LM. Time and frequency analysis of the static balance in young adults with Down syndrome. Gait Posture 2011; 33: 23-28.

21 Takara K, Gaspar M, Metring NL, Mateus-Vasconcelos ECL, Cliquet A, Abreu DCC. Evaluation of the influence of different types of seats on postural control in individuals with paraplegia. Spinal Cord 2010; 48: 825-827.

22 Adegoke B, Ogwumike O, Olatemiju A. Dynamic balance and level of lesion in spinal cord injured patients. Afr J Med Med Sci 2002; 31: 357-360. 